\title{
Gradient recovery for elliptic interface problem: II. immersed finite element methods
}

\author{
Hailong Guo ${ }^{\mathrm{a}}$, Xu Yang ${ }^{\mathrm{a}, *}$ \\ ${ }^{a}$ Department of Mathematics, University of California Santa Barbara, CA, 93106
}

\begin{abstract}
This is the second paper on the study of gradient recovery for elliptic interface problem. In our previous work [H. Guo and X. Yang, 2016, arXiv:1607.05898], we developed a novel gradient recovery technique for finite element method based on body-fitted mesh. In this paper, we propose new gradient recovery methods for two immersed interface finite element methods: symmetric and consistent immersed finite method [H. Ji, J. Chen and Z. Li, J. Sci. Comput., 61 (2014), 533-557] and Petrov-Galerkin immersed finite element method [T.Y. Hou, X.-H. Wu and Y. Zhang, Commun. Math. Sci., 2 (2004), 185-205, and S. Hou and X.-D. Liu, J. Comput. Phys., 202 (2005), 411-445]. Compared to body-fitted mesh based gradient recover methods, immersed finite element methods provide a uniform way of recovering gradient on regular meshes. Numerical examples are presented to confirm the superconvergence of both gradient recovery methods. Moreover, they provide asymptotically exact a posteriori error estimators for both immersed finite element methods.
\end{abstract}

Keywords: elliptic interface problem, immersed finite element method, gradient recovery, superconvergence, a posteriori error estimator

2010 MSC: 35R05, 65N30, 65N15

\section{Introduction}

We are interested in developing gradient recovery methods for the following elliptic interface problem

$$
\begin{aligned}
-\nabla \cdot(\beta(z) \nabla u(z)) & =f(z), & & z \text { in } \Omega \backslash \Gamma, \\
u & =0, & & z \text { on } \partial \Omega,
\end{aligned}
$$

where $\Omega$ is a bounded polygonal domain with Lipschitz boundary $\partial \Omega$ in $\mathbb{R}^{2}$, and $\Gamma$ is the interface which splits $\Omega$ into two disjoint subdomains $\Omega^{-}$and $\Omega^{+}$. Note that the interface $\Gamma$ can be given by a zero level of level set function $[32,37$.

\footnotetext{
* Corresponding author Yang)

Email addresses: hlguo@math.ucsb.edu (Hailong Guo), xuyang@math.ucsb.edu (Xu
}

Preprint submitted to Elsevier

January 3, 2017

(C) 2017. This manuscript version is made available under the Elsevier user license http://www.elsevier.com/open-access/userlicense/1.0/ 
The interface problem is characterized by the following piecewise smooth diffusion coefficient $\beta(z) \geq \beta_{0}$,

$$
\beta(z)= \begin{cases}\beta^{-}(z) & \text { if } z \in \Omega^{-} \\ \beta^{+}(z) & \text { if } z \in \Omega^{+}\end{cases}
$$

which has a finite jump of function value across the interface $\Gamma$. We consider homogeneous jump conditions at the interface $\Gamma$ as below,

$$
\begin{aligned}
{[u]_{\Gamma} } & =u^{+}-u^{-}=0, \\
{\left[\beta \partial_{n} u\right]_{\Gamma} } & =\beta^{+} u_{n}^{+}-\beta^{-} u_{n}^{-}=0,
\end{aligned}
$$

where $\partial_{n} u=\nabla u \cdot n$ denotes the normal flux with $n$ being the unit outer normal vector of the interface $\Gamma$.

Simulation of the interface problem (1.1)- 1.5 is an important problem in the fields of fluid dynamics and material science, where background is composed by rather different materials. Discontinuities of coefficients at interface lead to nonsmooth solutions in general, and thus raise a challenge for designing efficient numerical methods for (1.1)- 1.5 .

Two mainstreams of existing numerical methods for 1.1 - (1.5) are bodyfitted mesh-based methods and immersed boundary/interface methods. Bodyfitted mesh-based methods resolve discontinuities by generating mesh grids to align with interface, and then use standard finite element methods. This type of methods can provide high order accuracy, with nearly optimal error estimates established in, for example, [2, 4, 9, 40]. Despite its merit of accuracy, a main drawback of such methods is the requirement of a body-fitted mesh generator, which can be technically involved and time consuming especially when the geometry of interface becomes complicated. Therefore, it will be more convenient to develop numerical methods based unfitted mesh (e.g. Cartesian mesh). A rich literature can be found in this direction including immersed boundary method (IBM) by Peskin [34, 35] and immersed interface method (IIM) by Leveque and Li [25], just to name a few.

In IBM, Dirac $\delta$-function is used to model discontinuity and discretized to distribute a singular source to nearest grid point. In IIM, a special finite difference scheme is constructed near interface to get an accurate approximation of the solution. Moreover, IIM was also developed in the framework of finite element method [27, 26, 29]. Interested readers are referred to 28 for a review of this type of methods. In 29, $\mathrm{Li}$, Lin and $\mathrm{Wu}$ proposed a nonconforming immersed finite element method (IFEM) by modifying the basis functions on elements crossing interface. Chou etc. established optimal error estimates in $L^{2}$ and $H^{1}$ norms in [13. However, it only achieved first order (suboptimal) convergence in $L^{\infty}$ norm due to discontinuities of test functions. To overcome this drawback, Ji, Chen, and $\mathrm{Li}$ added a correction term into the bilinear for$\mathrm{m}$ of the nonconforming IFEM to penalize the discontinuities at interface [23], which showed optimal convergence rate in $L^{2}$ and $H^{1}$ norms. They also numerically verified that the method achieved second order convergence in $L^{\infty}$ norm. 
Another weak form formulation was derived in [20, 21, 22] based on PetrovGalerkin method for the discretization of elliptic interface problem, which has been numerically verified to have optimal convergence rate in $L^{2}, H^{1}$ and $L^{\infty}$ norms.

Superconvergence analysis of elliptic interface problem has been a challenging problem due to the of lack regularity of solution at interface. Standard gradient recovery methods [42, 43, 41, 30, 1, 18, only work well for elliptic problems with smooth coefficient. As far as we know, only limited work has been done in the development of gradient recovery methods for elliptic interface problem. For example, [11, 12 proposed two special interpolation formula to recover flux for linear and quadratic immersed finite element method in one-dimension. A more recent work 38 showed a supercloseness between finite element solution and linear interpolation of the true solution for linear finite element method based on body-fitted mesh. In our previous work [17, we developed an immerse polynomial preserving recovery (IPPR) method based on body-fitted mesh and proved its superconvergence for both mildly unstructured and adaptive refined meshes.

As a continuous study of [17, we propose new gradient recovery methods in this paper based on two immersed finite element methods: symmetric and consistent immersed finite element (SCIFEM) 23] and Petrov-Galerkin immersed finite element method (PGIFEM) 20, 21, 22. The development of the methods is based on the following two observations: firstly, the solution is piecewise smooth on each subdomain despite of its low global regularity; secondly, finite element solution is discontinuous at interface even though the exact solution is continuous. Accordingly, we design the gradient recovery methods by two steps: enriching and smoothing. We first define an enriching operator to enrich the discontinuous finite element solution into continuous one on a local body-fitted mesh obtained by adding extra nodes [29. Such type of enriching operator has been well studied for nonconforming finite element and plays an important role in a priori error estimates [16] and convergence analysis of multigrid methods [5, 6, 7. Then we apply the IPPR gradient recovery operator developed in [17. to the enriched finite element solution. We prove that the proposed gradient recovery operator is a bounded linear operator, and numerically verify that the recovered gradient is $\mathcal{O}\left(h^{1.5}\right)$ superconvergent to exact gradient. As a byproduct, we observe the $\mathcal{O}\left(h^{1.5}\right)$ supercloseness between finite element solution and linear interpolation of true solution for both SCIFEM 23] and PGIFEM [20, 21, 22].

The rest of the paper is organized as follows. In Section 2, we briefly review two immersed finite element methods, SCIFEM and PGIFEM, as a preparation for designing gradient recovery methods. In Section 3, we first define an enriching operator and prove several properties of the operator. Then, we propose the gradient recovery methods for SCIFEM and PGIFEM and prove that the gradient recovery operator is a linear, bounded and consistent operator. In Section 4, serval numerical examples are presented to confirm the superconvergence of the gradient recovery method. We make conclusive remarks in Section 5. 


\section{Review on immersed finite element methods}

In this section, we briefly review two immersed finite element methods, symmetric and consistent immersed finite element method [23] and Petrov-Galerkin immersed finite element method [20, 21, 22, based on which we shall develop superconvergent gradient recovery methods for elliptic interface problem (1.1)(1.5) in Section 3.

\subsection{Notations}

We first summarize the notations that will be used in this paper. We will use standard notations for Sobolev spaces and their associate norms given in [8, 14, 15]. For a subdomain $A$ of $\Omega$, let $\mathbb{P}_{m}(A)$ be the space of polynomials of degree less than or equal to $m$ in $A$ and $n_{m}$ be the dimension of $\mathbb{P}_{m}(A)$ which equals to $\frac{1}{2}(m+1)(m+2)$. $W^{k, p}(A)$ denotes the Sobolev space with norm $\|\cdot\|_{k, p, A}$ and seminorm $|\cdot|_{k, p, A}$. When $p=2, W^{k, 2}(A)$ is simply denoted by $H^{k}(A)$ and the subscript $p$ is omitted in its associate norm and seminorm. As in [38, denote $W^{k, p}\left(\Omega^{-} \cup \Omega^{+}\right)$as the function space consisting of piecewise Sobolev function $w$ such that $\left.w\right|_{\Omega^{-}} \in W^{k, p}\left(\Omega^{-}\right)$and $\left.w\right|_{\Omega^{+}} \in W^{k, p}\left(\Omega^{+}\right)$. For the function space $W^{k, p}\left(\Omega^{-} \cup \Omega^{+}\right)$, define its associated norm as

$$
\|w\|_{k, p, \Omega^{-} \cup \Omega^{+}}=\left(\|w\|_{k, p, \Omega^{-}}^{p}+\|w\|_{k, p, \Omega^{+}}\right)^{1 / p},
$$

and associated seminorm as

$$
|w|_{k, p, \Omega^{-} \cup \Omega^{+}}=\left(|w|_{k, p, \Omega^{-}}^{p}+|w|_{k, p, \Omega^{+}}\right)^{1 / p} .
$$

Let $C$ denote a generic positive constant which may be different at different occurrences. For the sake of simplicity, we use $x \lesssim y$ to mean that $x \leq C y$ for some constant $C$ independent of mesh size.

Without loss of generality, we simply suppose $\mathcal{T}_{h}$ is a uniform triangulation of $\Omega$ with $h=\operatorname{diam}(T)$. Assume $h$ is small enough so that the interface $\Gamma$ never crosses any edge of $\mathcal{T}_{h}$ more than two times. The elements of $\mathcal{T}_{h}$ can be divided into categories : regular element and interface element. We call an element $T$ interface element if the interface $\Gamma$ passes the interior of $T$; otherwise we call it regular element. Remark that if $\Gamma$ only passes two vertices of an element $T$, we treat the element $T$ as a regular element. Let $\mathcal{T}_{h}^{i}$ and $\mathcal{T}_{h}^{r}$ denote the set of all interface elements and regular elements respectively. The set of all vertices of $\mathcal{T}_{h}$ is denoted by $\mathcal{N}_{h}$.

\subsection{Variational formula}

The variational formulation to elliptic interface problem $(1.1)-(1.5)$ is given by finding $u \in H_{0}^{1}(\Omega)$ such that

$$
(\beta \nabla u, \nabla v)=(f, v), \quad \forall v \in H_{0}^{1}(\Omega),
$$


where $(\cdot, \cdot)$ is standard $L_{2}$-inner product in the spaces $L^{2}(\Omega)$. By the positiveness of $\beta$, Lax-Milgram Theorem implies (2.1) has a unique solution. 9, 36, proved that $u \in H^{r}\left(\Omega^{-} \cup \Omega^{+}\right)$for $0 \leq r \leq 2$ and

$$
\|u\|_{r, \Omega^{-} \cup \Omega^{+}} \lesssim\|f\|_{0, \Omega}+\|g\|_{r-3 / 2, \Gamma},
$$

if $f \in L^{2}(\Omega)$ and $g \in H^{r-3 / 2}(\Gamma)$.

\subsection{Immersed finite element methods}

The key idea of immersed interface methods is to construct special basis functions in interface elements to incorporate jump conditions (1.4) and 1.5). As an illustration, we consider a typical interface element $T$ as in Figure 1 . Let $z_{4}$ and $z_{5}$ be the intersection points between the interface $\Gamma$ and edges of the element. Connect the line segment $\overline{z_{4} z_{5}}$ and it forms an approximation of interface $\Gamma$ in the element $T$, denoted by $\left.\Gamma_{h}\right|_{T}$. Then the element $T$ is spitted into two parts: $T^{-}$and $T^{+}$. The special basis $\phi_{i}$ on the interface element $T$ is constructed as the following piecewise linear function

$$
\phi_{i}(z)= \begin{cases}\phi_{i}^{+}=a^{+}+b^{+} x+c^{+} y, & z=(x, y) \in T^{+}, \\ \phi_{i}^{-}=a^{-}+b^{-} x+c^{-} y, & z=(x, y) \in T^{-},\end{cases}
$$

where the coefficients are determined by the following linear system

$$
\begin{aligned}
& \phi_{i}\left(z_{1}\right)=\delta_{i 1}, \phi_{i}\left(z_{2}\right)=\delta_{i 2}, \phi_{i}\left(z_{3}\right)=\delta_{i 3}, \\
& \phi_{i}^{+}\left(z_{4}\right)=\phi_{i}^{-}\left(z_{4}\right), \phi_{i}^{+}\left(z_{5}\right)=\phi_{i}^{-}\left(z_{5}\right), \beta^{+} \partial_{n} \phi_{i}^{+}=\beta^{-} \partial_{n} \phi_{i}^{-},
\end{aligned}
$$

for $i=1,2,3$. The immersed finite element space $V_{h}[29$ is defined as

$$
\begin{aligned}
& V_{h}:=\left\{v \in V_{h}:\left.v\right|_{T} \in V_{h}(T) \text { and } v \text { is continuous on } \mathcal{N}_{h},\right\}, \\
& V_{h, 0}=\left\{v \in V_{h}: v(z)=0 \text { for all } z \in \mathcal{N}_{h} \cap \partial \Omega\right\},
\end{aligned}
$$

where

$$
V_{h}(T):= \begin{cases}\left\{v \mid v \in \mathbb{P}_{1}(T)\right\}, & \text { if } T \in \mathcal{T}_{h}^{r} \\ \{v \mid v \text { is defined by } 2.3-2.5\}, & \text { if } T \in \mathcal{T}_{h}^{i} .\end{cases}
$$

Note that in general $V_{h}$ is a nonconforming finite element space and 26] shows it has optimal approximation capability.

\subsubsection{Symmetric and consistent immersed finite element method}

Let $\mathcal{E}_{h}$ denote the set of all edges in $\mathcal{T}_{h}$, and then $\mathcal{E}_{h}$ consists of interface edge $\mathcal{E}_{h}^{i}$ and regular edge $\mathcal{E}_{h}^{r}$, defined by

$$
\mathcal{E}_{h}^{i}=\left\{e \in \mathcal{E}_{h}: \stackrel{\circ}{\cap} \Gamma \neq \emptyset\right\},, \mathcal{E}_{h}^{r}=\mathcal{E}_{h} \backslash \mathcal{E}_{h}^{i} .
$$

For any interior edge $e$, there exist two triangles $T_{1}$ and $T_{2}$ such that $T_{1} \cap T_{2}=e$. Denote $n_{e}$ as the unit normal of $e$ pointing from $T_{1}$ to $T_{2}$, and define

$$
\begin{aligned}
& \{\nabla u\}=\frac{1}{2}\left(\left.\nabla u\right|_{T_{1}}+\left.\nabla u\right|_{T_{2}}\right), \\
& {[u]=\left.u\right|_{T_{1}}-\left.u\right|_{T_{2}} .}
\end{aligned}
$$




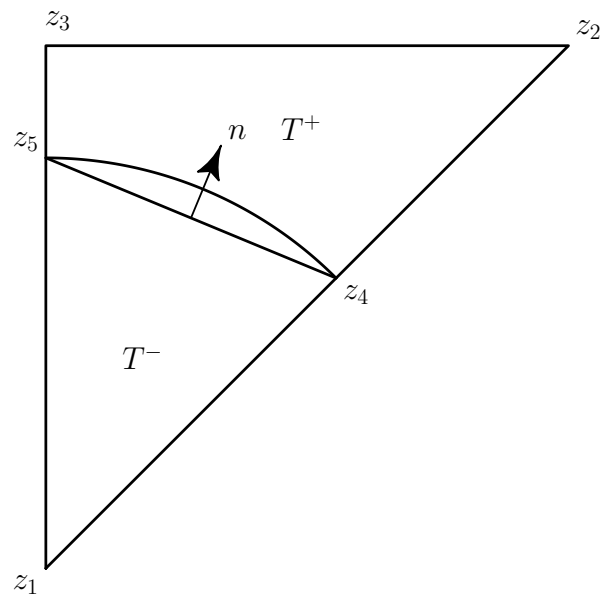

Figure 1: Typical example of interface element.

The symmetric and consistent immersed finite element method (SCIFEM) 23 . seeks $u_{h}^{s c} \in V_{h, 0}$ such that

$$
a_{h}^{s c}\left(u_{h}^{s c}, v_{h}\right)=\left(f, v_{h}\right), \quad \forall v_{h} \in V_{h, 0},
$$

where

$$
a_{h}^{s c}(u, v)=\sum_{T \in \mathcal{T}_{h}} \int_{T} \beta \nabla u \cdot \nabla v d x+\sum_{e \in \mathcal{E}_{h}^{i}} \int_{e}(\{\beta \nabla u\}[u]+\{\beta \nabla v\}[u]) \cdot n_{e} d s
$$

In 23], Ji, Chen, and Li showed the bilinear form (2.13) was consist and numerically verified its coercivity. Moreover, 23 proved the following convergence results:

Theorem 2.1. Let $u$ be the solution of 1.1)-(1.5) and $u_{h}$ be the solution of 2.12. Then the following error estimates hold:

$$
\begin{aligned}
& \left(\sum_{T \in \mathcal{T}_{h}}\left|u-u_{h}^{s c}\right|_{H^{1}(T)}^{2}\right)^{1 / 2} \lesssim h\|u\|_{2, \Omega^{+} \cup \Omega^{-}}, \\
& \left\|u-u_{h}^{s c}\right\|_{0, \Omega} \lesssim h\|u\|_{2, \Omega^{+} \cup \Omega^{-}} .
\end{aligned}
$$

Remark 2.2. The main difference between SCIFEM and classical immersed finite element method 29] is that the bilinear form of SCIFEM 2.13) contains one more term to penalize the discontinuous of basis function at the intersecting points of interface and edge. Numerical results in 23 , show that SCIFEM has $O\left(h^{2}\right)$ convergence in $L^{\infty}$-norm. 


\subsubsection{Petrov-Galerkin immersed finite element method}

Denote the standard $C^{0}$ linear finite element space on $\mathcal{T}_{h}$ by $S_{h}$ and $S_{h, 0}=$ $S_{h} \cap H_{0}^{1}(\Omega)$. Then the Petrov-Galerkin immersed finite element method (PGIFEM) [22, 20, 21] is to find $u_{h}^{p g} \in V_{h, 0}$ such that

$$
a_{h}\left(u_{h}^{p g}, v_{h}\right)=\left(f, v_{h}\right), \quad \forall v_{h} \in S_{h, 0},
$$

where

$$
a_{h}(u, v)=\sum_{T \in \mathcal{T}_{h}} \int_{T} \beta \nabla u \cdot \nabla v d x .
$$

Remark 2.3. To our best knowledge, there has been no analytical results on estimating PGIFEM, however, plenty of numerical simulations indicate that it can achieve optimal convergence rate in both $L_{2}, H_{1}$ and $L_{\infty}$ norms $[22,20,21$.

\section{Gradient recovery for immersed finite element methods}

In the section, we systematically introduce gradient recovery methods for SCIFEM and PGIFEM reviewed in last section. We first define an enriching operator, and then apply the immersed polynomial preserving recovery operator [17] to the enriched finite element solution.

\subsection{Enriching operator}

To define the enriching operator, one needs to generate a local body-fitted mesh $\widehat{\mathcal{T}}_{h}$ based on $\mathcal{T}_{h}$ by adding new vertices into $\mathcal{N}_{h}$ which divides interface element into three subtriangles. Then the new triangulation is constructed as below [29]:

1. Keep all regular elements unchanged.

2. For each interface element $T$, split it into a small triangle and a quadrilateral by connecting two intersection points, and then divide the quadrilateral into two subtriangles by an auxiliary line connecting a vertex and an intersection point. The choice of auxiliary line is made so that there at least exists one angle between $\frac{\pi}{4}$ and $\frac{3 \pi}{4}$ in the two new subtriangles.

Remark 3.1. Note that the new triangulation can contain narrow triangles, and thus standard linear finite element method deteriorates on $\widehat{\mathcal{T}}_{h}$. However, the propose of introducing the body-fitted mesh $\widehat{\mathcal{T}}_{h}$ is just for enriching existing immersed finite element solution instead of solving interface problem directly on it.

Let $X_{h}$ be the $C^{0}$ linear finite element space defined on $\widehat{\mathcal{T}}_{h}$. We construct an enriching operator $E_{h}: V_{h} \rightarrow X_{h}$ by averaging the discontinuous values at intersection points. Let $\widehat{\mathcal{N}}_{h}$ denote all vertices in $\widehat{\mathcal{T}}_{h}$, and one has $\mathcal{N}_{h} \subset \widehat{\mathcal{N}}_{h}$. For

any $z \in \widehat{\mathcal{N}}_{h}$, let $\widehat{\mathcal{T}}_{z}$ denote the set of all triangles in $\widehat{\mathcal{T}}_{h}$ having $z$ as their vertex and define

$$
\left(E_{h} v\right)(z)=\frac{1}{\left|\widehat{\mathcal{T}}_{z}\right|} \sum_{\widehat{T} \in \widehat{\mathcal{T}}_{z}} v_{\widehat{T}}(z),
$$


with $\left|\widehat{\mathcal{T}}_{z}\right|$ being the cardinality of $\widehat{\mathcal{T}}_{z}$ and $v_{\widehat{T}}=\left.v\right|_{\widehat{T}}$. We can define $E_{h} v$ on $\Omega$ by standard linear finite element interpolation in $X_{h}$ after obtaining the values $\left(E_{h} v\right)(z)$ at all vertices. It is easy to see that $\left(E_{h} v\right)(z)=v(z)$ for all $z \in \widehat{\mathcal{N}}_{h} \cap \mathcal{N}_{h}$, which means $\left(E_{h} v\right)(z)=v(z)$ for all $z \in \widehat{\mathcal{N}}_{h}$ provided that $v$ is continuous.

Remark 3.2. The purpose of the enriching operator is to make the discontinuous immersed finite element solution become continuous as the true solution.

For the enriching operator $E_{h}$, we can prove the following error estimate.

Theorem 3.3. For any $v \in V_{h}$, one has

$$
\sum_{T \in \widehat{\mathcal{T}}_{h}}\left\|E_{h} v-v\right\|_{0, T}^{2} \lesssim h^{2} \sum_{T \in \mathcal{T}_{h}}|v|_{1, T}^{2} .
$$

Proof. For any $z \in \widehat{\mathcal{N}}_{h} \backslash \mathcal{N}_{h}$, there exists an $e \in \mathcal{E}_{h}^{i}$ so that $z \in e$. Let $T_{1}$ and $T_{2}$ be the two triangles in $\mathcal{T}_{h}$ so that $T_{1} \cap T_{2}=e$. Then $\widehat{T} \subset T_{1}$ or $\widehat{T} \subset T_{2}$ for any $\widehat{T} \in \widehat{\mathcal{T}}_{z}$. Hence $v_{\widehat{T}}(z)=v_{T_{1}}(z)$ or $v_{\widehat{T}}(z)=v_{T_{2}}(z)$. Then for any $\widehat{T}_{a}, \widehat{T}_{b} \in \widehat{\mathcal{T}}_{z}$, we can find $p \in \mathcal{N}_{h}$ such that

$$
\begin{aligned}
& {\left[v_{\widehat{T}_{a}}(z)-v_{\widehat{T}_{b}}(z)\right]^{2} } \\
\leq & {\left[v_{T_{1}}(z)-v_{T_{2}}(z)\right]^{2} } \\
\leq & {\left[v_{T_{1}}(z)-v_{T_{1}}(p)\right]^{2}+\left[v_{T_{1}}(p)-v_{T_{2}}(p)\right]^{2}+\left[v_{T_{2}}(p)-v_{T_{2}}(z)\right]^{2} } \\
= & {\left[v_{T_{1}}(z)-v_{T_{1}}(p)\right]^{2}+\left[v_{T_{2}}(p)-v_{T_{2}}(z)\right]^{2} } \\
\lesssim & |v|_{1, T_{1} \cup T_{2}}^{2},
\end{aligned}
$$

where we have used the fact that $v_{T_{1}}(p)=v_{T_{2}}(p)$ since $p \in \mathcal{N}_{h}$ in the first equality and the mean value theorem [7] in the last inequality.

Combining (3.1) and (3.3) gives, for any $\widehat{T} \in \widehat{\mathcal{T}}_{z}$,

$$
\left[\left(E_{h} v-v_{\widehat{T}}\right)(z)\right]^{2} \lesssim|v|_{1, T_{1} \cup T_{2}}^{2}, \forall v \in V_{h},
$$

which implies that

$$
\begin{aligned}
\left\|E_{h} v-v\right\|_{0, \widehat{T}}^{2} & \leq|\widehat{T}| \sum_{z \in \mathcal{N}(\widehat{T})}\left[\left(E_{h} v-v_{\widehat{T}}\right)(z)\right]^{2} \\
& \lesssim h^{2} \sum_{T \in \mathcal{T}(\widehat{T})}|v|_{1, T}^{2},
\end{aligned}
$$

where $\mathcal{T}(\widehat{T})=\left\{T \in \mathcal{T}_{h}: T \cap \widehat{T} \neq \emptyset\right\}$. Taking summation over all $\widehat{\mathcal{T}}_{h}$ produces the inequality 3.2 .

Corollary 3.4. For any $v \in V_{h}$, we have

$$
\begin{aligned}
& \left\|E_{h} v\right\|_{0, \Omega} \lesssim\|v\|_{0, \Omega}, \\
& \left|E_{h} v\right|_{1, \Omega} \lesssim|v|_{1, \Omega} .
\end{aligned}
$$


Proof. We first prove the inequality (3.6). Notice that

$$
\begin{aligned}
\left\|E_{h} v\right\|_{0, \Omega} & \lesssim\left\|E_{h} v-v\right\|_{0, \Omega}+\|v\|_{0, \Omega} \\
& \lesssim h\|\nabla v\|_{0, \Omega}+\|v\|_{0, \Omega} \\
& \lesssim\|v\|_{0, \Omega},
\end{aligned}
$$

where we have used the standard inverse estimate [14, 8, in the last inequality. Using (3.2) and standard inverse estimate yields

$$
\begin{aligned}
\left|E_{h} v\right|_{1, \Omega} & \leq\left|E_{h} v-v\right|_{1, \Omega}+|v|_{1, \Omega} \\
& \lesssim h^{-1}\left\|E_{h} v-v\right\|_{0, \Omega}+|v|_{1, \Omega} \\
& \lesssim|v|_{1, \Omega},
\end{aligned}
$$

which completes our proof.

\subsection{Gradient Recovery Operator}

The edges of $\widehat{\mathcal{T}}_{h}$ with both ending points lying on $\Gamma$ form an approximation of the interface $\Gamma$, denoted by $\Gamma_{h}$, then the triangulation $\widehat{\mathcal{T}}_{h}$ is divided into the following two disjoint sets by $\Gamma_{h}$ :

$$
\begin{aligned}
& \widehat{\mathcal{T}}_{h}^{-}:=\left\{T \in \widehat{\mathcal{T}}_{h} \mid \text { all three vertices of } T \text { are in } \overline{\Omega^{-}}\right\}, \\
& \widehat{\mathcal{T}}_{h}^{+}:=\left\{T \in \widehat{\mathcal{T}}_{h} \mid \text { all three vertices of } T \text { are in } \overline{\Omega^{+}}\right\} .
\end{aligned}
$$

Suppose $X_{h}^{-}$and $X_{h}^{+}$are the continuous linear finite element spaces defined on $\widehat{\mathcal{T}}_{h}^{-}$and $\widehat{\mathcal{T}}_{h}^{+}$respectively.

Let $G_{h}^{I}: X_{h} \rightarrow\left(X_{h}^{-} \cup X_{h}^{+}\right) \times\left(X_{h}^{-} \cup X_{h}^{+}\right)$be the immersed polynomial preserving recovery (IPPR) operator introduced in [17. Let $u_{h}$ be the solution of either symmetric and consistent immersed finite element method or PetrovGalerkin immersed finite element method. The recovered gradient of $u_{h}$ is defined as

$$
R_{h} u_{h}=G_{h}^{I}\left(E_{h} u_{h}\right) .
$$

Remark 3.5. The proposed gradient recovery method consists of two steps: firstly, we enrich the immersed finite element solution by the enriching operator; then we recover the gradient of the enriched solution.

Remark 3.6. The gradient recovery method require doing a least-squares fitting at very vertex of $\mathcal{T}_{h}$ with computation cost of order $\mathcal{O}(1)$. Hence, the total computational cost of recovery procedure is of order $\mathcal{O}(N)$. It can be ignored compared to the cost of solving original problem.

It is easy to see that $R_{h}$ is a linear operator from $V_{h}$ to $\left(X_{h}^{-} \cup X_{h}^{+}\right) \times\left(X_{h}^{-} \cup\right.$ $X_{h}^{+}$), and one can prove the following boundedness results.

Theorem 3.7. Denote $R_{h}$ to be the recovered operator defined in 3.12 , and then

$$
\left\|R_{h} u_{h}\right\|_{0, \Omega^{-} \cup \Omega^{+}} \lesssim\left|u_{h}\right|_{1, h}
$$


Proof. By the definition of IPPR recovery operator in [17, we have

$$
\left\|R_{h} u_{h}\right\|_{0, \Omega^{-}}=\left\|G_{h}^{I} E_{h} u_{h}\right\|_{0, \Omega^{-}} \lesssim\left|E_{h} u_{h}\right|_{1, \Omega^{-}},
$$

and

$$
\left\|R_{h} u_{h}\right\|_{0, \Omega^{+}}=\left\|G_{h}^{I} E_{h} u_{h}\right\|_{0, \Omega^{+}} \lesssim\left|E_{h} u_{h}\right|_{1, \Omega^{+}} .
$$

Then the estimate follows by that

$$
\begin{aligned}
\left\|R_{h} u_{h}\right\|_{0, \Omega^{-} \cup \Omega^{+}} & \leq\left\|R_{h} u_{h}\right\|_{0, \Omega^{-}}+\left\|R_{h} u_{h}\right\|_{0, \Omega^{-}} \\
& \lesssim\left|E_{h} u_{h}\right|_{1, \Omega^{-}}+\left|E_{h} u_{h}\right|_{1, \Omega^{+}} \\
& \lesssim\left|E_{h} u_{h}\right|_{1, \Omega} \\
& \lesssim\left|u_{h}\right|_{1, \Omega},
\end{aligned}
$$

where we have used Corollary 3.4

Theorem 3.7 implies $R_{h}$ is a linear bounded operator. Moreover, we have the following consistency result:

Theorem 3.8. Let $R_{h}: V_{h} \rightarrow\left(X_{h}^{-} \cup X_{h}^{+}\right) \times\left(X_{h}^{-} \cup X_{h}^{+}\right)$be the gradient recovery operator defined in 3.12 . Given $u \in H^{3}\left(\Omega^{-} \cup \Omega^{+}\right) \cap C^{0}(\Omega)$, one has

$$
\left\|R_{h} u_{I}-\nabla u\right\|_{0, \Omega} \lesssim h^{2}\|u\|_{3, \Omega^{-} \cup \Omega^{+}},
$$

where $u_{I}$ is interpolation of $u$ into linear finite element space $X_{h}$.

Proof. Since $u \in C^{0}(\Omega)$, one has that $u_{I} \in C^{0}(\Omega)$ and then $E_{h} u_{I}=u_{I}$. Therefore, we have $R_{h} u_{I}=G_{h}^{I} E_{h} u_{I}=G_{h} u_{I}$. Theorem 3.6 in [17] implies that

$$
\left\|R_{h} u_{I}-\nabla u\right\|_{0, \Omega}=\left\|G_{h}^{I} u_{I}-\nabla u\right\|_{0, \Omega} \leq h^{2}\|u\|_{3, \Omega^{-} \cup \Omega^{+}},
$$

which completes our proof.

Remark 3.9. Theorem 3.8 implies $R_{h}$ is consistent. In addition, it is a local gradient recovery operator. Therefore, $R_{h}$ satisfies the three conditions of a good gradient recovery operator described in [1, and should serve as an ideal candidate of gradient recovery operator for both SCIFEM and PGIFEM.

Remark 3.10. One of the most practical applications of gradient recovery techniques is to construct asymptotically exact a posteriori error estimators [1, 3, 19, 31, 42, 43, for adaptive computational methods. Based on the recovery operator $R_{h}$, one can define a local a posteriori error estimator on element $T \in \mathcal{T}_{h}$ as

$$
\eta_{T}= \begin{cases}\left\|\beta^{1 / 2}\left(R_{h} u_{h}-\nabla u_{h}\right)\right\|_{0, T}, & \text { if } T \in \mathcal{T}_{h}^{r}, \\ \left(\sum_{\widehat{T} \subset T, \widehat{T} \in \widehat{T}_{h}}\left\|\beta^{1 / 2}\left(R_{h} u_{h}-\nabla u_{h}\right)\right\|_{0, \widehat{T}}^{2}\right)^{\frac{1}{2}}, & \text { if } T \in \mathcal{T}_{h}^{i},\end{cases}
$$

and the corresponding global error estimator as

$$
\eta_{h}=\left(\sum_{T \in \mathcal{T}_{h}} \eta_{T}^{2}\right)^{1 / 2}
$$


which provides an asymptotically exact a posteriori error estimator for SCIFEM and PGIFEM. The readers are referred to [10, 39] for residual-type a posteriori error estimator for immersed finite element methods.

\section{Numerical Results}

In the section, we give several numerical examples to verify the superconvergence of gradient recovery methods for both SCIFEM and PGIFEM. The computational domain of the first four examples are chosen as $\Omega=[-1,1] \times[-1,1]$. The uniform triangulation of $\Omega$ is obtained by dividing $\Omega$ into $N^{2}$ subsquares and then dividing each subsquare into two right triangles. In the first four tests, we take $N=2^{k}$ with $k=5,6,7,8,9,10,11$. In the lastp example, we consider a nonlinear interface problem with homogeneous jump conditions on an annulus domain. For convenience, we shall use the following error norms in all examples:

$$
D e:=\left\|u-u_{h}\right\|_{1, \Omega}, \quad D^{i} e:=\left\|\nabla u_{I}-\nabla u_{h}\right\|_{0, \Omega}, \quad D^{r} e:=\left\|\nabla u-R_{h} u_{h}\right\|_{0, \Omega} .
$$

Example 4.1. In this example, we consider the elliptic interface problem (1.1) with a circular interface of radius $r_{0}=0.6$ as studied in [29]. The exact solution is

$$
u(z)= \begin{cases}\frac{r^{3}}{\beta^{-}} & \text {if } z \in \Omega_{-}, \\ \frac{r^{3}}{\beta^{+}}+\left(\frac{1}{\beta^{-}}-\frac{1}{\beta^{+}}\right) r_{0}^{3} & \text { if } z \in \Omega^{+}\end{cases}
$$

where $r=\sqrt{x^{2}+y^{2}}$.

Tables 16 show the numerical results of both SCIFEM and PGIFEM with three typical different jump ratios: $\beta^{-} / \beta^{+}=1 / 10$ (moderate jump), $\beta^{-} / \beta^{+}=$ $1 / 1000$ (large jump), and $\beta^{-} / \beta^{+}=1000$ (large jump). In all different cases, optimal $\mathcal{O}(h)$ convergence can be observed for $H^{1}$-semi error of finite element solution, which consists with the numerical results in $[23,20,22$. The recovered gradient superconverges to the exact gradient at a rate of $\mathcal{O}\left(h^{1.5}\right)$. Moreover, we numerically observe the supercloseness between gradient of the finite element solution and its finite element interpolation for both SCIFEM and PGIFEM; see column 5 of Tables 1,6 .

Table 1: Numerical results of SCIFEM for Example 4.1 with $\beta^{+}=10, \beta^{-}=1$.

\begin{tabular}{|c|c|c|c|c|c|c|}
\hline$N$ & $D e$ & order & $D^{i} e$ & order & $D_{r}^{r} e$ & order \\
\hline \hline 32 & $5.71 \mathrm{e}-02$ & - & $1.47 \mathrm{e}-02$ & - & $2.19 \mathrm{e}-02$ & - \\
\hline 64 & $2.94 \mathrm{e}-02$ & 0.96 & $4.48 \mathrm{e}-03$ & 1.72 & $7.48 \mathrm{e}-03$ & 1.55 \\
\hline 128 & $1.47 \mathrm{e}-02$ & 1.00 & $1.84 \mathrm{e}-03$ & 1.28 & $2.31 \mathrm{e}-03$ & 1.69 \\
\hline 256 & $7.38 \mathrm{e}-03$ & 0.99 & $6.46 \mathrm{e}-04$ & 1.51 & $7.40 \mathrm{e}-04$ & 1.64 \\
\hline 512 & $3.70 \mathrm{e}-03$ & 1.00 & $2.36 \mathrm{e}-04$ & 1.45 & $2.93 \mathrm{e}-04$ & 1.34 \\
\hline 1024 & $1.85 \mathrm{e}-03$ & 1.00 & $8.11 \mathrm{e}-05$ & 1.54 & $1.02 \mathrm{e}-04$ & 1.52 \\
\hline 2048 & $9.26 \mathrm{e}-04$ & 1.00 & $2.83 \mathrm{e}-05$ & 1.52 & $3.44 \mathrm{e}-05$ & 1.57 \\
\hline
\end{tabular}


Table 2: Numerical results of PGIFEM for Example 4.1 with $\beta^{+}=10, \beta^{-}=1$.

\begin{tabular}{|c|c|c|c|c|c|c|}
\hline$N$ & $D e$ & order & $D^{i} e$ & order & $D_{r}^{r} e$ & order \\
\hline \hline 32 & $5.92 \mathrm{e}-02$ & - & $2.15 \mathrm{e}-02$ & - & $3.09 \mathrm{e}-02$ & - \\
\hline 64 & $2.98 \mathrm{e}-02$ & 0.99 & $6.61 \mathrm{e}-03$ & 1.71 & $1.01 \mathrm{e}-02$ & 1.61 \\
\hline 128 & $1.48 \mathrm{e}-02$ & 1.01 & $2.59 \mathrm{e}-03$ & 1.35 & $3.33 \mathrm{e}-03$ & 1.61 \\
\hline 256 & $7.41 \mathrm{e}-03$ & 1.00 & $9.00 \mathrm{e}-04$ & 1.53 & $1.08 \mathrm{e}-03$ & 1.63 \\
\hline 512 & $3.71 \mathrm{e}-03$ & 1.00 & $3.27 \mathrm{e}-04$ & 1.46 & $4.11 \mathrm{e}-04$ & 1.39 \\
\hline 1024 & $1.85 \mathrm{e}-03$ & 1.00 & $1.13 \mathrm{e}-04$ & 1.54 & $1.43 \mathrm{e}-04$ & 1.52 \\
\hline 2048 & $9.26 \mathrm{e}-04$ & 1.00 & $3.99 \mathrm{e}-05$ & 1.50 & $4.92 \mathrm{e}-05$ & 1.54 \\
\hline
\end{tabular}

Table 3: Numerical results of SCIFEM for Example 4.1 with $\beta^{+}=1000, \beta^{-}=1$.

\begin{tabular}{|c|c|c|c|c|c|c|}
\hline$N$ & $D e$ & order & $D^{i} e$ & order & $D_{r}^{r} e$ & order \\
\hline \hline 32 & $5.69 \mathrm{e}-02$ & - & $2.43 \mathrm{e}-02$ & - & $2.46 \mathrm{e}-02$ & - \\
\hline 64 & $2.77 \mathrm{e}-02$ & 1.04 & $3.50 \mathrm{e}-03$ & 2.79 & $6.44 \mathrm{e}-03$ & 1.93 \\
\hline 128 & $1.38 \mathrm{e}-02$ & 1.00 & $1.61 \mathrm{e}-03$ & 1.12 & $1.95 \mathrm{e}-03$ & 1.72 \\
\hline 256 & $6.95 \mathrm{e}-03$ & 0.99 & $5.36 \mathrm{e}-04$ & 1.58 & $6.34 \mathrm{e}-04$ & 1.62 \\
\hline 512 & $3.49 \mathrm{e}-03$ & 1.00 & $1.95 \mathrm{e}-04$ & 1.46 & $2.54 \mathrm{e}-04$ & 1.32 \\
\hline 1024 & $1.75 \mathrm{e}-03$ & 1.00 & $6.61 \mathrm{e}-05$ & 1.56 & $8.76 \mathrm{e}-05$ & 1.53 \\
\hline 2048 & $8.74 \mathrm{e}-04$ & 1.00 & $2.29 \mathrm{e}-05$ & 1.53 & $2.98 \mathrm{e}-05$ & 1.55 \\
\hline
\end{tabular}

Table 4: Numerical results of PGIFEM for Example 4.1 with $\beta^{+}=1000, \beta^{-}=1$.

\begin{tabular}{|c|c|c|c|c|c|c|}
\hline$N$ & $D e$ & order & $D^{i} e$ & order & $D_{r}^{r} e$ & order \\
\hline \hline 32 & $5.95 \mathrm{e}-02$ & - & $3.27 \mathrm{e}-02$ & - & $4.55 \mathrm{e}-02$ & - \\
\hline 64 & $2.91 \mathrm{e}-02$ & 1.03 & $9.35 \mathrm{e}-03$ & 1.81 & $1.21 \mathrm{e}-02$ & 1.91 \\
\hline 128 & $1.44 \mathrm{e}-02$ & 1.02 & $4.03 \mathrm{e}-03$ & 1.21 & $4.54 \mathrm{e}-03$ & 1.41 \\
\hline 256 & $7.10 \mathrm{e}-03$ & 1.02 & $1.45 \mathrm{e}-03$ & 1.48 & $1.48 \mathrm{e}-03$ & 1.62 \\
\hline 512 & $3.53 \mathrm{e}-03$ & 1.01 & $5.68 \mathrm{e}-04$ & 1.35 & $5.94 \mathrm{e}-04$ & 1.31 \\
\hline 1024 & $1.76 \mathrm{e}-03$ & 1.01 & $1.90 \mathrm{e}-04$ & 1.58 & $1.95 \mathrm{e}-04$ & 1.60 \\
\hline 2048 & $8.76 \mathrm{e}-04$ & 1.00 & $6.80 \mathrm{e}-05$ & 1.48 & $6.96 \mathrm{e}-05$ & 1.49 \\
\hline
\end{tabular}

Table 5: Numerical results of SCIFEM for Example 4.1 with $\beta^{+}=1, \beta^{-}=1000$.

\begin{tabular}{|c|c|c|c|c|c|c|}
\hline$N$ & $D e$ & order & $D^{i} e$ & order & $D_{r}^{r} e$ & order \\
\hline \hline 32 & $1.95 \mathrm{e}-01$ & - & $1.35 \mathrm{e}-02$ & - & $1.92 \mathrm{e}-02$ & - \\
\hline 64 & $9.79 \mathrm{e}-02$ & 1.00 & $3.60 \mathrm{e}-03$ & 1.91 & $8.14 \mathrm{e}-03$ & 1.24 \\
\hline 128 & $4.90 \mathrm{e}-02$ & 1.00 & $1.48 \mathrm{e}-03$ & 1.28 & $2.21 \mathrm{e}-03$ & 1.88 \\
\hline 256 & $2.45 \mathrm{e}-02$ & 1.00 & $5.56 \mathrm{e}-04$ & 1.42 & $7.46 \mathrm{e}-04$ & 1.57 \\
\hline 512 & $1.23 \mathrm{e}-02$ & 1.00 & $1.81 \mathrm{e}-04$ & 1.61 & $2.38 \mathrm{e}-04$ & 1.65 \\
\hline 1024 & $6.13 \mathrm{e}-03$ & 1.00 & $6.44 \mathrm{e}-05$ & 1.49 & $8.57 \mathrm{e}-05$ & 1.48 \\
\hline 2048 & $3.06 \mathrm{e}-03$ & 1.00 & $2.33 \mathrm{e}-05$ & 1.47 & $2.99 \mathrm{e}-05$ & 1.52 \\
\hline
\end{tabular}

Example 4.2. In this example, we consider the elliptic interface problem 
Table 6: Numerical results of PGIFEM for Example 4.1 with $\beta^{+}=1, \beta^{-}=1000$.

\begin{tabular}{|c|c|c|c|c|c|c|}
\hline$N$ & $D e$ & order & $D^{i} e$ & order & $D_{r}^{r} e$ & order \\
\hline \hline 32 & $5.95 \mathrm{e}-02$ & - & $3.27 \mathrm{e}-02$ & - & $4.55 \mathrm{e}-02$ & - \\
\hline 64 & $2.91 \mathrm{e}-02$ & 1.03 & $9.35 \mathrm{e}-03$ & 1.81 & $1.21 \mathrm{e}-02$ & 1.91 \\
\hline 128 & $1.44 \mathrm{e}-02$ & 1.02 & $4.03 \mathrm{e}-03$ & 1.21 & $4.54 \mathrm{e}-03$ & 1.41 \\
\hline 256 & $7.10 \mathrm{e}-03$ & 1.02 & $1.45 \mathrm{e}-03$ & 1.48 & $1.48 \mathrm{e}-03$ & 1.62 \\
\hline 512 & $3.53 \mathrm{e}-03$ & 1.01 & $5.68 \mathrm{e}-04$ & 1.35 & $5.94 \mathrm{e}-04$ & 1.31 \\
\hline 1024 & $1.76 \mathrm{e}-03$ & 1.01 & $1.90 \mathrm{e}-04$ & 1.58 & $1.95 \mathrm{e}-04$ & 1.60 \\
\hline 2048 & $8.76 \mathrm{e}-04$ & 1.00 & $6.80 \mathrm{e}-05$ & 1.48 & $6.96 \mathrm{e}-05$ & 1.49 \\
\hline
\end{tabular}

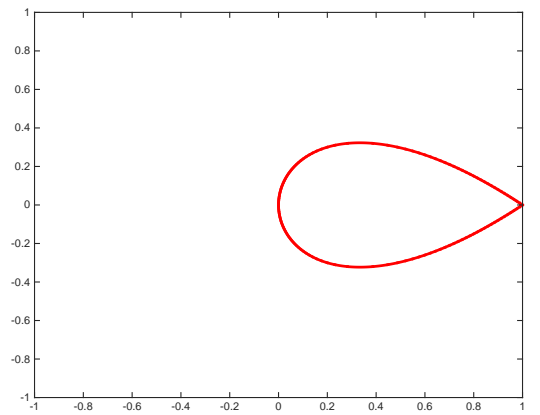

(a)

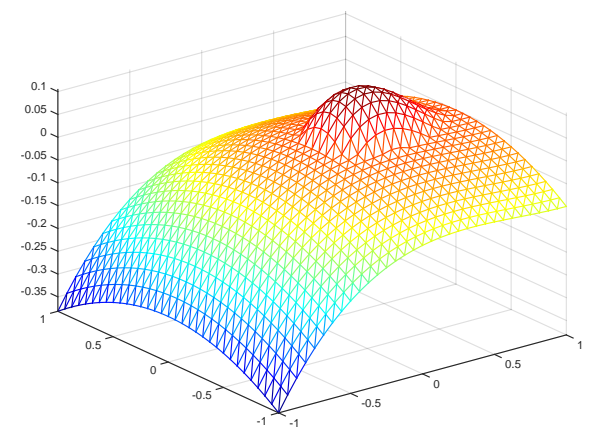

(b)

Figure 2: Example 2 with $\beta^{+}=10, \beta^{-}=1$ : (a) Shape of interface; (b) Numerical solution of PGIFEM on the coarsest mesh used in Table 8.

Table 7: Numerical results of SCIFEM for Example 4.2.

\begin{tabular}{|c|c|c|c|c|c|c|}
\hline$N$ & $D e$ & order & $D^{i} e$ & order & $D_{r}^{r} e$ & order \\
\hline \hline 32 & $3.04 \mathrm{e}-02$ & - & $7.01 \mathrm{e}-03$ & - & $1.12 \mathrm{e}-02$ & - \\
\hline 64 & $1.54 \mathrm{e}-02$ & 0.98 & $4.63 \mathrm{e}-03$ & 0.60 & $4.16 \mathrm{e}-03$ & 1.42 \\
\hline 128 & $7.44 \mathrm{e}-03$ & 1.05 & $8.71 \mathrm{e}-04$ & 2.41 & $1.02 \mathrm{e}-03$ & 2.03 \\
\hline 256 & $3.71 \mathrm{e}-03$ & 1.01 & $3.55 \mathrm{e}-04$ & 1.30 & $4.28 \mathrm{e}-04$ & 1.25 \\
\hline 512 & $1.85 \mathrm{e}-03$ & 1.00 & $1.27 \mathrm{e}-04$ & 1.49 & $1.52 \mathrm{e}-04$ & 1.49 \\
\hline 1024 & $9.24 \mathrm{e}-04$ & 1.00 & $4.31 \mathrm{e}-05$ & 1.56 & $5.50 \mathrm{e}-05$ & 1.47 \\
\hline 2048 & $4.62 \mathrm{e}-04$ & 1.00 & $1.55 \mathrm{e}-05$ & 1.48 & $1.99 \mathrm{e}-05$ & 1.47 \\
\hline
\end{tabular}

(1.1) with shape edge as in 23, 24. The level set function of the interface is $\phi=-y^{2}+((x-1) \tan (\theta))^{2} x$ with $\theta$ being a parameter. The interface is displayed in Figure 2(a) The right hand function $f$ is chosen to fit the exact solution $u(x, y)=\phi(x, y) / \beta$.

Numerically we test the case $\beta^{-}=1$ and $\beta^{+}=1000$ when $\theta=40$. The corresponding numerical results are shown in Tables 7 and 8 , from which one can see that $D e$ decays at a optimal rate of $\mathcal{O}(h)$, while $D^{i} e$ and $D^{r} e$ tend 


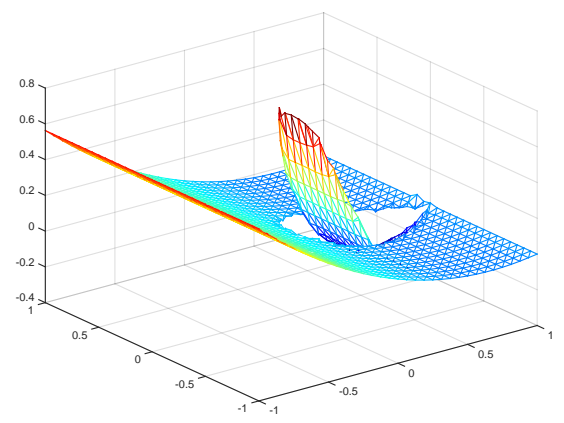

(a)

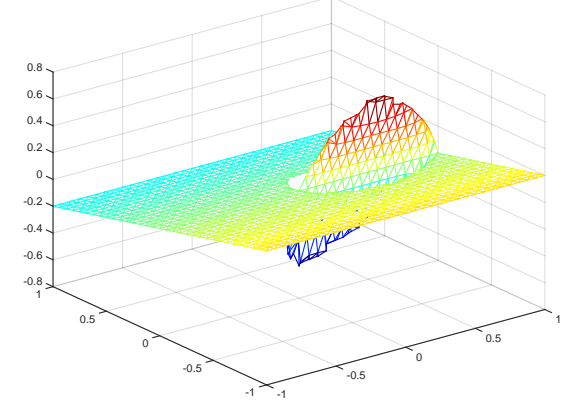

(b)

Figure 3: Plots of recovered gradient based on PGIFEM for Example 4.2 with $\beta^{+}=10, \beta^{-}=1$ : (a) $x$-component; (b) $y$-component.

Table 8: Numerical results of PGIFEM for Example 4.2.

\begin{tabular}{|c|c|c|c|c|c|c|}
\hline$N$ & $D e$ & order & $D^{i} e$ & order & $D_{r}^{r} e$ & order \\
\hline \hline 32 & $3.57 \mathrm{e}-02$ & - & $1.98 \mathrm{e}-02$ & - & $2.09 \mathrm{e}-02$ & - \\
\hline 64 & $1.70 \mathrm{e}-02$ & 1.08 & $8.37 \mathrm{e}-03$ & 1.24 & $8.09 \mathrm{e}-03$ & 1.37 \\
\hline 128 & $7.96 \mathrm{e}-03$ & 1.09 & $2.95 \mathrm{e}-03$ & 1.50 & $2.73 \mathrm{e}-03$ & 1.57 \\
\hline 256 & $3.82 \mathrm{e}-03$ & 1.06 & $9.97 \mathrm{e}-04$ & 1.57 & $9.41 \mathrm{e}-04$ & 1.54 \\
\hline 512 & $1.88 \mathrm{e}-03$ & 1.02 & $3.72 \mathrm{e}-04$ & 1.42 & $3.54 \mathrm{e}-04$ & 1.41 \\
\hline 1024 & $9.32 \mathrm{e}-04$ & 1.02 & $1.29 \mathrm{e}-04$ & 1.53 & $1.24 \mathrm{e}-04$ & 1.51 \\
\hline 2048 & $4.64 \mathrm{e}-04$ & 1.01 & $4.57 \mathrm{e}-05$ & 1.50 & $4.31 \mathrm{e}-05$ & 1.52 \\
\hline
\end{tabular}

to zero at a superconvergent rate of $\mathcal{O}\left(h^{1.5}\right)$. Figure 2(b) plots the numerical solution of PGIFEM on the coarsest mesh and Figure 3 shows the recovered gradient.

Table 9: Numerical results of SCIFEM for Example 4.3.

\begin{tabular}{|c|c|c|c|c|c|c|}
\hline$N$ & $D e$ & order & $D^{i} e$ & order & $D_{r}^{r} e$ & order \\
\hline \hline 32 & $1.19 \mathrm{e}+00$ & - & $1.61 \mathrm{e}-01$ & - & $1.93 \mathrm{e}-01$ & - \\
\hline 64 & $5.93 \mathrm{e}-01$ & 1.00 & $5.98 \mathrm{e}-02$ & 1.43 & $7.24 \mathrm{e}-02$ & 1.42 \\
\hline 128 & $2.96 \mathrm{e}-01$ & 1.00 & $2.14 \mathrm{e}-02$ & 1.48 & $2.69 \mathrm{e}-02$ & 1.43 \\
\hline 256 & $1.48 \mathrm{e}-01$ & 1.00 & $7.80 \mathrm{e}-03$ & 1.46 & $9.66 \mathrm{e}-03$ & 1.48 \\
\hline 512 & $7.41 \mathrm{e}-02$ & 1.00 & $2.75 \mathrm{e}-03$ & 1.50 & $3.49 \mathrm{e}-03$ & 1.47 \\
\hline 1024 & $3.70 \mathrm{e}-02$ & 1.00 & $9.84 \mathrm{e}-04$ & 1.48 & $1.23 \mathrm{e}-03$ & 1.51 \\
\hline 2048 & $1.85 \mathrm{e}-02$ & 1.00 & $3.49 \mathrm{e}-04$ & 1.50 & $4.37 \mathrm{e}-04$ & 1.49 \\
\hline
\end{tabular}

Example 4.3. In the example, we consider the elliptic interface problem (1.1) with ellipse interface given by the zero level set of the function $\phi(x, y)=$ $\frac{x^{2}}{0.5^{2}}+\frac{y^{2}}{0.25^{2}}-1$ as studied in [23, 24]. Here, we choose the case of variable 
Table 10: Numerical results of PGIFEM for Example 4.3.

\begin{tabular}{|c|c|c|c|c|c|c|}
\hline$N$ & $D e$ & order & $D^{i} e$ & order & $D_{r}^{r} e$ & order \\
\hline \hline 32 & $1.19 \mathrm{e}+00$ & - & $1.55 \mathrm{e}-01$ & - & $1.89 \mathrm{e}-01$ & - \\
\hline 64 & $5.93 \mathrm{e}-01$ & 1.00 & $5.81 \mathrm{e}-02$ & 1.42 & $7.19 \mathrm{e}-02$ & 1.39 \\
\hline 128 & $2.96 \mathrm{e}-01$ & 1.00 & $2.09 \mathrm{e}-02$ & 1.48 & $2.66 \mathrm{e}-02$ & 1.43 \\
\hline 256 & $1.48 \mathrm{e}-01$ & 1.00 & $7.61 \mathrm{e}-03$ & 1.45 & $9.56 \mathrm{e}-03$ & 1.48 \\
\hline 512 & $7.41 \mathrm{e}-02$ & 1.00 & $2.68 \mathrm{e}-03$ & 1.50 & $3.45 \mathrm{e}-03$ & 1.47 \\
\hline 1024 & $3.70 \mathrm{e}-02$ & 1.00 & $9.61 \mathrm{e}-04$ & 1.48 & $1.22 \mathrm{e}-03$ & 1.51 \\
\hline 2048 & $1.85 \mathrm{e}-02$ & 1.00 & $3.41 \mathrm{e}-04$ & 1.50 & $4.33 \mathrm{e}-04$ & 1.49 \\
\hline
\end{tabular}

coefficient $\beta(x, y)$ as

$$
\beta(x, y)= \begin{cases}1+0.5\left(x^{2}-x y+y^{2}\right) & \text { if }(x, y) \in \Omega^{-}, \\ 1 & \text { if }(x, y) \in \Omega^{+} .\end{cases}
$$

The right hand side function $f$ and boundary condition are given by the exact solution $u(x, y)=\phi(x, y) / \beta(x, y)$.

Tables 9 and 10 list the numerical errors, which provide a verification of the $\mathcal{O}(h)$ convergence for semi- $H^{1}$ error, and $\mathcal{O}\left(h^{1.5}\right)$ supercloseness and superconvergence.

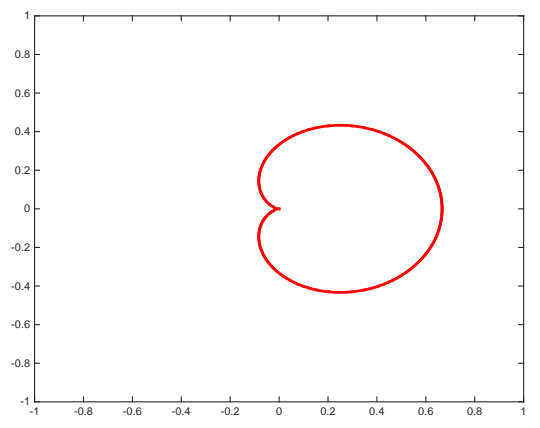

(a)

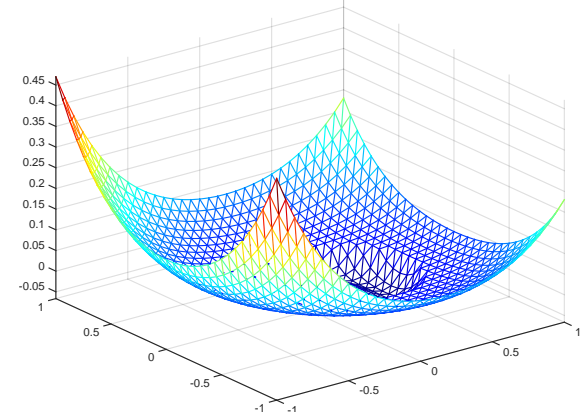

(b)

Figure 4: Example 4.4 with $\beta^{+}=10, \beta^{-}=1$ : (a) Shape of interface; (b) Numerical solution of PGIFEM on the coarsest mesh used in Table 12 .

Example 4.4. In this example, we consider the interface problem 1.1 with a cardioid interface as in [20]. The interface curve $\Gamma$ is the zero level of the function

$$
\phi(x, y)=\left(3\left(x^{2}+y^{2}\right)-x\right)^{2}-x^{2}-y^{2},
$$

as shown Figure 4(a). We choose the exact solution $u(x, y)=\phi(x, y) / \beta(x, y)$, where

$$
\beta(x, y)= \begin{cases}x y+3 & \text { if }(x, y) \in \Omega^{-}, \\ 100 & \text { if }(x, y) \in \Omega^{+} .\end{cases}
$$




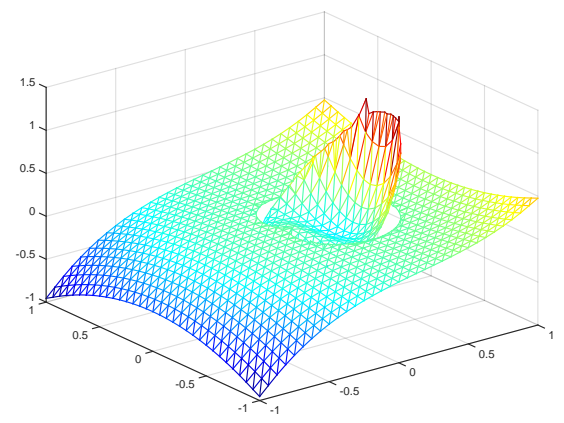

(a)

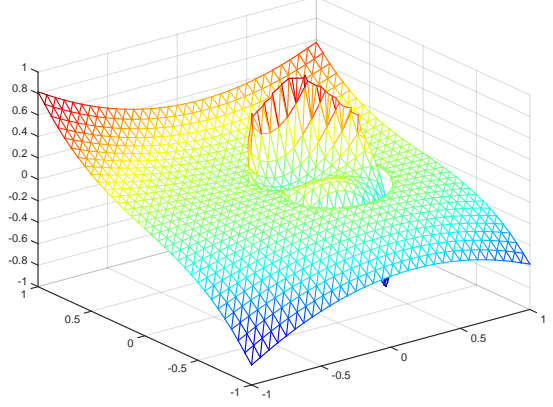

(b)

Figure 5: Plots of recovered gradient based on PGIFEM for Example 4.4 with $\beta^{+}=10, \beta^{-}=1$ : (a) $x$-component; (b) $y$-component.

Table 11: Numerical results of SCIFEM for Example 4.4.

\begin{tabular}{|c|c|c|c|c|c|c|}
\hline$N$ & $D e$ & order & $D^{i} e$ & order & $D_{r}^{r} e$ & order \\
\hline \hline 32 & $5.59 \mathrm{e}-02$ & - & $9.84 \mathrm{e}-03$ & - & $2.51 \mathrm{e}-02$ & - \\
\hline 64 & $2.88 \mathrm{e}-02$ & 0.96 & $4.09 \mathrm{e}-03$ & 1.27 & $7.98 \mathrm{e}-03$ & 1.65 \\
\hline 128 & $1.47 \mathrm{e}-02$ & 0.98 & $1.57 \mathrm{e}-03$ & 1.38 & $2.30 \mathrm{e}-03$ & 1.80 \\
\hline 256 & $7.39 \mathrm{e}-03$ & 0.99 & $5.72 \mathrm{e}-04$ & 1.46 & $7.21 \mathrm{e}-04$ & 1.67 \\
\hline 512 & $3.71 \mathrm{e}-03$ & 0.99 & $2.05 \mathrm{e}-04$ & 1.48 & $2.41 \mathrm{e}-04$ & 1.58 \\
\hline 1024 & $1.86 \mathrm{e}-03$ & 1.00 & $7.25 \mathrm{e}-05$ & 1.50 & $8.99 \mathrm{e}-05$ & 1.42 \\
\hline 2048 & $9.31 \mathrm{e}-04$ & 1.00 & $2.54 \mathrm{e}-05$ & 1.51 & $3.15 \mathrm{e}-05$ & 1.51 \\
\hline
\end{tabular}

Table 12: Numerical results of PGIFEM for Example 4.4.

\begin{tabular}{|c|c|c|c|c|c|c|}
\hline$N$ & $D e$ & order & $D^{i} e$ & order & $D_{r}^{r} e$ & order \\
\hline \hline 32 & $6.09 \mathrm{e}-02$ & - & $2.48 \mathrm{e}-02$ & - & $4.06 \mathrm{e}-02$ & - \\
\hline 64 & $3.01 \mathrm{e}-02$ & 1.01 & $9.06 \mathrm{e}-03$ & 1.45 & $1.36 \mathrm{e}-02$ & 1.58 \\
\hline 128 & $1.50 \mathrm{e}-02$ & 1.01 & $3.32 \mathrm{e}-03$ & 1.45 & $4.25 \mathrm{e}-03$ & 1.68 \\
\hline 256 & $7.48 \mathrm{e}-03$ & 1.00 & $1.16 \mathrm{e}-03$ & 1.51 & $1.45 \mathrm{e}-03$ & 1.55 \\
\hline 512 & $3.73 \mathrm{e}-03$ & 1.00 & $4.13 \mathrm{e}-04$ & 1.49 & $4.87 \mathrm{e}-04$ & 1.57 \\
\hline 1024 & $1.87 \mathrm{e}-03$ & 1.00 & $1.44 \mathrm{e}-04$ & 1.52 & $1.74 \mathrm{e}-04$ & 1.49 \\
\hline 2048 & $9.32 \mathrm{e}-04$ & 1.00 & $5.11 \mathrm{e}-05$ & 1.49 & $6.11 \mathrm{e}-05$ & 1.51 \\
\hline
\end{tabular}

As pointed in [20, the difficulty of the problem is that the interface is not even Lipschitz-continuous and has a singular point at the origin. Figure 4(b) plots the numerical solution of PGIFEM and Figure 5 shows the recovered gradient. The numerical errors are given in Tables 11 and 12, from which, one can also observe the optimal convergence and superconvergence for both SCIFEM and PGIFEM even though the interface is not Lipschitz-continuous.

Example 4.5. In this example, we consider the following nonlinear interface 
problem

$$
-\nabla \cdot(\beta(z) \nabla u(z))+u^{2}=f(z), \quad z \text { in } \Omega \backslash \Gamma,
$$

with homogeneous jump conditions 1.4 and 1.5 on an annulus domain $\Omega=$ $\{z: 1<|z|<3\}$. The interface curve $\Gamma$ is a circle centered at origin with the radius $r_{0}=2$. The exact solution is

$$
u(z)= \begin{cases}\frac{\log (r)}{\beta^{-}}, & \text {if } z \in \Omega_{-}, \\ \frac{\log (r)}{\beta^{+}}+\left(\frac{1}{\beta^{-}}-\frac{1}{\beta^{+}}\right) \log (2), & \text { if } z \in \Omega^{+},\end{cases}
$$

where $r=\sqrt{x^{2}+y^{2}}$. The right hand side function $f$ and boundary condition are determined by the exact solution.

The initial non body-fitted unstructured meshes are generated by DistMesh 33. The discretized nonlinear problems are solved by Newton's method. Tables 13 and 14 show the numerical results for SCIFEM and PGIFEM with $\beta^{-} / \beta^{+}=1 / 1000$ respectively. Note Dof $\approx h^{-2}$ for a two dimensional grid, the corresponding convergent rates with respect to the mesh size $h$ are twice as many as what we present in the Tables 13 and 14 . We can observe the same superconvergence and supercloseness results as linear problems on uniform meshes.

Table 13: Numerical results of SCIFEM for Example 4.5.

\begin{tabular}{|c|c|c|c|c|c|c|}
\hline Dof & $D e$ & order & $D^{i} e$ & order & $D_{r}^{r} e$ & order \\
\hline \hline 459 & $1.77 \mathrm{e}-01$ & - & $2.01 \mathrm{e}-02$ & - & $7.41 \mathrm{e}-02$ & - \\
\hline 1737 & $8.58 \mathrm{e}-02$ & 0.55 & $6.38 \mathrm{e}-03$ & 0.86 & $1.44 \mathrm{e}-02$ & 1.23 \\
\hline 6750 & $4.31 \mathrm{e}-02$ & 0.51 & $2.37 \mathrm{e}-03$ & 0.73 & $3.79 \mathrm{e}-03$ & 0.99 \\
\hline 26604 & $2.14 \mathrm{e}-02$ & 0.51 & $8.12 \mathrm{e}-04$ & 0.78 & $1.11 \mathrm{e}-03$ & 0.89 \\
\hline 105624 & $1.06 \mathrm{e}-02$ & 0.51 & $2.82 \mathrm{e}-04$ & 0.77 & $3.55 \mathrm{e}-04$ & 0.83 \\
\hline 420912 & $5.31 \mathrm{e}-03$ & 0.50 & $9.71 \mathrm{e}-05$ & 0.77 & $1.24 \mathrm{e}-04$ & 0.76 \\
\hline 1680480 & $2.65 \mathrm{e}-03$ & 0.50 & $3.46 \mathrm{e}-05$ & 0.75 & $4.33 \mathrm{e}-05$ & 0.76 \\
\hline
\end{tabular}

Table 14: Numerical results of PGIFEM for Example 4.5.

\begin{tabular}{|c|c|c|c|c|c|c|}
\hline Dof & $D e$ & order & $D^{i} e$ & order & $D_{r}^{r} e$ & order \\
\hline \hline 459 & $1.83 \mathrm{e}-01$ & - & $4.40 \mathrm{e}-02$ & - & $6.99 \mathrm{e}-02$ & - \\
\hline 1737 & $8.73 \mathrm{e}-02$ & 0.56 & $1.58 \mathrm{e}-02$ & 0.77 & $1.43 \mathrm{e}-02$ & 1.19 \\
\hline 6750 & $4.35 \mathrm{e}-02$ & 0.51 & $5.61 \mathrm{e}-03$ & 0.76 & $4.27 \mathrm{e}-03$ & 0.89 \\
\hline 26604 & $2.15 \mathrm{e}-02$ & 0.51 & $1.98 \mathrm{e}-03$ & 0.76 & $1.35 \mathrm{e}-03$ & 0.84 \\
\hline 105624 & $1.07 \mathrm{e}-02$ & 0.51 & $7.21 \mathrm{e}-04$ & 0.73 & $4.75 \mathrm{e}-04$ & 0.76 \\
\hline 420912 & $5.32 \mathrm{e}-03$ & 0.50 & $2.52 \mathrm{e}-04$ & 0.76 & $1.63 \mathrm{e}-04$ & 0.77 \\
\hline 1680480 & $2.66 \mathrm{e}-03$ & 0.50 & $8.96 \mathrm{e}-05$ & 0.75 & $5.65 \mathrm{e}-05$ & 0.77 \\
\hline
\end{tabular}

\section{Conclusion}

In this paper, we develop gradient recovery methods for both symmetric consistent immersed finite method and Petrov-Galerkin immersed finite element 
method. Theoretically, we prove that the proposed gradient recovery operator has consistency, localization, and boundedness properties. The superconvergence of recovered gradient is confirmed by five numerical examples using both piecewise constant and piecewise variable diffusion coefficients. Moreover, we numerically observe the supercloseness between immersed finite element solution and the linear interpolation of exact solution. Compared to body-fitted mesh-based gradient recovery methods, the proposed gradient recovery methods provide a uniform way of recovering gradient on regular meshes. One of our ongoing research project is to provide a theoretic justification for the observed superconvergence and supercloseness phenomenon

\section{Acknowledgement}

This work was partially supported by the NSF grant DMS-1418936, KI-Net NSF RNMS grant 1107291, and Hellman Family Foundation Faculty Fellowship, UC Santa Barbara. Part of work was done during the visit of both authors to

Beijing Computational Science Research Center, and we really appreciate their hospitality.

\section{References}

[1] Mark Ainsworth and J. Tinsley Oden. A posteriori error estimation in finite element analysis. Pure and Applied Mathematics (New York). WileyInterscience [John Wiley \& Sons], New York, 2000. ISBN 0-471-29411-X.

[2] Ivo Babuška. The finite element method for elliptic equations with discontinuous coefficients. Computing (Arch. Elektron. Rechnen), 5:207-213, 1970.

[3] Ivo Babuška and Theofanis Strouboulis. The finite element method and its reliability. Numerical Mathematics and Scientific Computation. The Clarendon Press, Oxford University Press, New York, 2001. ISBN 0-19850276-1.

[4] James H. Bramble and J. Thomas King. A finite element method for interface problems in domains with smooth boundaries and interfaces. $A d v$. Comput. Math., 6(2):109-138 (1997), 1996. ISSN 1019-7168.

[5] Susanne C. Brenner. Two-level additive Schwarz preconditioners for nonconforming finite element methods. Math. Comp., 65(215):897-921, 1996. ISSN 0025-5718.

[6] Susanne C. Brenner. Convergence of nonconforming multigrid methods without full elliptic regularity. Math. Comp., 68(225):25-53, 1999. ISSN 0025-5718.

[7] Susanne C. Brenner. Poincaré-Friedrichs inequalities for piecewise $H^{1}$ functions. SIAM J. Numer. Anal., 41(1):306-324, 2003. ISSN 0036-1429. 
[8] Susanne C. Brenner and L. Ridgway Scott. The mathematical theory of finite element methods, volume 15 of Texts in Applied Mathematics. Springer, New York, third edition, 2008. ISBN 978-0-387-75933-3.

[9] Zhiming Chen and Jun Zou. Finite element methods and their convergence for elliptic and parabolic interface problems. Numer. Math., 79(2):175-202, 1998. ISSN 0029-599X.

[10] Zhiming Chen, Yuanming Xiao, and Linbo Zhang. The adaptive immersed interface finite element method for elliptic and Maxwell interface problems. J. Comput. Phys., 228(14):5000-5019, 2009. ISSN 0021-9991.

[11] So-Hsiang Chou. An immersed linear finite element method with interface flux capturing recovery. Discrete Contin. Dyn. Syst. Ser. B, 17(7):23432357, 2012. ISSN 1531-3492.

[12] So-Hsiang Chou and C ATTANAYAKE. Flux recovery and superconvergence of quadratic immersed interface finite elements, DEC 2015.

[13] So-Hsiang Chou, Do Y. Kwak, and K. T. Wee. Optimal convergence analysis of an immersed interface finite element method. Adv. Comput. Math., 33(2):149-168, 2010. ISSN 1019-7168.

[14] Philippe G. Ciarlet. The finite element method for elliptic problems, volume 40 of Classics in Applied Mathematics. Society for Industrial and Applied Mathematics (SIAM), Philadelphia, PA, 2002. ISBN 0-89871-5148. Reprint of the 1978 original [North-Holland, Amsterdam; MR0520174 (58 \#25001)].

[15] Lawrence C. Evans. Partial differential equations, volume 19 of Graduate Studies in Mathematics. American Mathematical Society, Providence, RI, second edition, 2010. ISBN 978-0-8218-4974-3.

[16] Thirupathi Gudi. A new error analysis for discontinuous finite element methods for linear elliptic problems. Math. Comp., 79(272):2169-2189, 2010. ISSN 0025-5718.

[17] Hailong Guo and Xu Yang. Gradient recovery for elliptic interface problem: I. body-fitted mesh, 2016. arXiv:1607.05898 [math.NA].

[18] Hailong Guo and Zhimin Zhang. Gradient recovery for the CrouzeixRaviart element. J. Sci. Comput., 64(2):456-476, 2015. ISSN 0885-7474.

[19] Hailong Guo, Zhimin Zhang, and Ren Zhao. Superconvergent two-grid methods for elliptic eigenvalue problems. J. Sci. Comput., pages 1-24, 2016. ISSN 0885-7474. doi: 10.1007/s10915-016-0245-2.

[20] Songming Hou and Xu-Dong Liu. A numerical method for solving variable coefficient elliptic equation with interfaces. J. Comput. Phys., 202(2):411445, 2005. ISSN 0021-9991. 
[21] Songming Hou, Peng Song, Liqun Wang, and Hongkai Zhao. A weak formulation for solving elliptic interface problems without body fitted grid. $J$. Comput. Phys., 249:80-95, 2013. ISSN 0021-9991.

[22] Thomas Y. Hou, Xiao-Hui Wu, and Yu Zhang. Removing the cell resonance error in the multiscale finite element method via a Petrov-Galerkin formulation. Commun. Math. Sci., 2(2):185-205, 2004. ISSN 1539-6746.

[23] Haifeng Ji, Jinru Chen, and Zhilin Li. A symmetric and consistent immersed finite element method for interface problems. J. Sci. Comput., 61 (3):533-557, 2014. ISSN 0885-7474.

[24] Do Y. Kwak, Kye T. Wee, and Kwang S. Chang. An analysis of a broken $P_{1}$-nonconforming finite element method for interface problems. SIAM J. Numer. Anal., 48(6):2117-2134, 2010. ISSN 0036-1429.

[25] Randall J. LeVeque and Zhi Lin Li. The immersed interface method for elliptic equations with discontinuous coefficients and singular sources. SIAM J. Numer. Anal., 31(4):1019-1044, 1994. ISSN 0036-1429.

[26] Z. Li, T. Lin, Y. Lin, and R. C. Rogers. An immersed finite element space and its approximation capability. Numer. Methods Partial Differential Equations, 20(3):338-367, 2004. ISSN 0749-159X.

[27] Zhilin Li. The immersed interface method using a finite element formulation. Appl. Numer. Math., 27(3):253-267, 1998. ISSN 0168-9274.

[28] Zhilin Li and Kazufumi Ito. The immersed interface method, volume 33 of Frontiers in Applied Mathematics. Society for Industrial and Applied Mathematics (SIAM), Philadelphia, PA, 2006. ISBN 0-89871-609-8. Numerical solutions of PDEs involving interfaces and irregular domains.

[29] Zhilin Li, Tao Lin, and Xiaohui Wu. New Cartesian grid methods for interface problems using the finite element formulation. Numer. Math., 96 (1):61-98, 2003. ISSN 0029-599X.

[30] A. Naga and Z. Zhang. The polynomial-preserving recovery for higher order finite element methods in 2D and 3D. Discrete Contin. Dyn. Syst. Ser. B, 5(3):769-798, 2005. ISSN 1531-3492.

[31] Ahmed Naga and Zhimin Zhang. A posteriori error estimates based on the polynomial preserving recovery. SIAM J. Numer. Anal., 42(4):1780-1800 (electronic), 2004. ISSN 0036-1429.

[32] Stanley Osher and Ronald Fedkiw. Level set methods and dynamic implicit surfaces, volume 153 of Applied Mathematical Sciences. Springer-Verlag, New York, 2003. ISBN 0-387-95482-1.

[33] Per-Olof Persson and Gilbert Strang. A simple mesh generator in Matlab. SIAM Rev., 46(2):329-345 (electronic), 2004. ISSN 0036-1445. 
[34] Charles S. Peskin. Numerical analysis of blood flow in the heart. J. Computational Phys., 25(3):220-252, 1977. ISSN 0021-9991.

[35] Charles S. Peskin. The immersed boundary method. Acta Numer., 11: 479-517, 2002. ISSN 0962-4929.

[36] Ja. A. Roütberg and Z. G. Šeftel'. A theorem on homeomorphisms for elliptic systems and its applications. Mathematics of the USSR-Sbornik, 78 (3):439-465, 1969.

[37] J. A. Sethian. Level set methods, volume 3 of Cambridge Monographs on Applied and Computational Mathematics. Cambridge University Press, Cambridge, 1996. ISBN 0-521-57202-9. Evolving interfaces in geometry, fluid mechanics, computer vision, and materials science.

[38] Huayi Wei, Long Chen, Yunqing Huang, and Bin Zheng. Adaptive mesh refinement and superconvergence for two-dimensional interface problems. SIAM J. Sci. Comput., 36(4):A1478-A1499, 2014. ISSN 1064-8275.

[39] Chin-Tien Wu, Zhilin Li, and Ming-Chih Lai. Adaptive mesh refinement for elliptic interface problems using the non-conforming immersed finite element method. Int. J. Numer. Anal. Model., 8(3):466-483, 2011. ISSN 1705-5105.

[40] Jinchao Xu. Error estimates of the finite element method for the 2nd order elliptic equations with discontinuous coefficients. J. Xiangtan Univ., 1:1-5, 1982.

[41] Zhimin Zhang and Ahmed Naga. A new finite element gradient recovery method: superconvergence property. SIAM J. Sci. Comput., 26(4):11921213 (electronic), 2005. ISSN 1064-8275.

[42] O. C. Zienkiewicz and J. Z. Zhu. The superconvergent patch recovery and a posteriori error estimates. I. The recovery technique. Internat. J. Numer. Methods Engrg., 33(7):1331-1364, 1992. ISSN 0029-5981.

[43] O. C. Zienkiewicz and J. Z. Zhu. The superconvergent patch recovery and a posteriori error estimates. II. Error estimates and adaptivity. Internat. J. Numer. Methods Engrg., 33(7):1365-1382, 1992. ISSN 0029-5981. 